\title{
Curvature of the pseudocritical line in QCD: Taylor expansion matches analytic continuation
}

\author{
Claudio Bonati, ${ }^{1,2, *}$ Massimo D’Elia, ${ }^{1,2, \dagger}$ Francesco Negro, ${ }^{2, \ddagger}$ Francesco Sanfilippo, ${ }^{3, \S}$ and Kevin Zambello, ${ }^{4, \|}$ \\ ${ }^{1}$ Università di Pisa, Largo B. Pontecorvo 3, I-56127 Pisa, Italy \\ ${ }^{2}$ INFN Sezione di Pisa, Largo B. Pontecorvo 3, I-56127 Pisa, Italy \\ ${ }^{3}$ INFN Sezione di Roma Tre, Via della Vasca Navale 84, I-00146 Roma, Italy \\ ${ }^{4}$ Università di Parma and INFN, Gruppo Collegato di Parma, \\ Parco Area delle Scienze 7/A, I-43124 Parma, Italy
}

(Received 11 June 2018; published 17 September 2018)

\begin{abstract}
We determine the curvature of the pseudocritical line of $N_{f}=2+1$ QCD with physical quark masses via Taylor expansion in the quark chemical potentials. We adopt a discretization based on stout improved staggered fermions and the tree level Symanzik gauge action; the location of the pseudocritical temperature is based on chiral symmetry restoration. Simulations are performed on lattices with different temporal extent $\left(N_{t}=6,8,10\right)$, leading to a continuum extrapolated curvature $\kappa=0.0145(25)$, which is in very good agreement with the continuum extrapolation obtained via analytic continuation and the same discretization, $\kappa=0.0135(20)$. This result eliminates the possible tension emerging when comparing analytic continuation with earlier results obtained via Taylor expansion.
\end{abstract}

DOI: $10.1103 /$ PhysRevD.98.054510

\section{INTRODUCTION}

The exploration of the QCD phase diagram is a subject of continuous experimental and theoretical investigation. One of the main issues is represented by the determination of the pseudocritical line in the $T-\mu_{B}$ plane separating the low- $T$ phase, characterized by color confinement and chiral symmetry breaking, from the high- $T$ phase where the socalled quark-gluon plasma (QGP) is thought to be realized. Lattice QCD simulations at nonzero $\mu_{B}$ are still hindered by the well-known sign problem; however, various methods are already effective to circumvent the problem at least for small $\mu_{B}$, where the pseudocritical line can be approximated at the lowest order in a Taylor expansion in $\mu_{B}^{2}$,

$$
\frac{T_{c}\left(\mu_{B}\right)}{T_{c}}=1-\kappa\left(\frac{\mu_{B}}{T_{c}}\right)^{2}+O\left(\mu_{B}^{4}\right)
$$

The curvature $\kappa$ of the pseudocritical line has been determined on the lattice both by analytic continuation [1-12],

\footnotetext{
*claudio.bonati@df.unipi.it

massimo.delia@unipi.it

fnegro@pi.infn.it

§sanfilippo@ roma3.infn.it

"kevin.zambello@pr.infn.it
}

Published by the American Physical Society under the terms of the Creative Commons Attribution 4.0 International license. Further distribution of this work must maintain attribution to the author(s) and the published article's title, journal citation, and DOI. Funded by SCOAP. exploiting results obtained at imaginary chemical potentials, and by Taylor expansion [13-16], i.e., by suitable combinations of expectation values determined at zero chemical potential. The pseudocritical line for small values of $\mu_{B}$ has been investigated also by continuum approaches to the QCD phase diagram (see, e.g., Refs. [17-21]).

Recently, various lattice investigations have led to a determination of $\kappa$ by analytic continuation for QCD with physical or almost physical quark masses [22-26]. In particular, Refs. $[24,25]$ have provided continuum extrapolated values for $\kappa$ which are, respectively, $\kappa=0.0135(20)$ and $\kappa=0.0149(21)$. The two studies adopted a similar discretization (stout improved staggered fermions and the tree level Symanzik gauge action) and slightly different setups for the quark chemical potentials: in Ref. [25], the strangeness neutrality condition reproduced in the heavyion experimental environment was enforced explicitly by tuning the strange quark chemical potential $\mu_{s}$ appropriately; in Ref. [24], instead, $\mu_{s}$ was set to zero while checking at the same time that its influence on $\kappa$ is negligible (see also Ref. [27]). Results obtained by analytic continuation but adopting a different lattice discretization (HISQ staggered fermions) have led to similar results $[22,26]$.

Such results are typically larger than earlier results obtained via Taylor expansion [28,29], reporting $\kappa \sim 0.006$. In particular, Ref. [29] reported a continuum extrapolated value $\kappa=$ $0.0066(20)$ adopting the same discretization and the same observables (chiral condensate) as in Refs. [23,24], i.e., a value which is more than two standard deviations away from the result from analytic continuation. As discussed in 
Ref. [23], only a small part of this discrepancy can be accounted for by the different prescriptions used to determine the dependence of $T_{c}$ on $\mu_{B}$, so that a tension remains.

The agreement between results obtained by the two methods is a necessary requirement in order to state that one has a full control over all systematics involved in analytic continuation and in Taylor expansion. Therefore, the importance of clarifying any possible tension cannot be overestimated; indeed, efforts in this direction are already in progress, for instance by adopting the HISQ staggered discretization [27].

In this study, we present a new continuum extrapolation for the curvature obtained via Taylor expansion, considering the same stout staggered discretization adopted in Refs. [24,29]. In particular, we consider different prescriptions to determine $\kappa$ via Taylor expansion and the analysis of the renormalized condensate: for fixed $N_{t}=6$, we show that they provide consistent results; then, exploiting simulations on different values of $N_{t}\left(N_{t}=6,8,10\right)$, we are able to provide results extrapolated to the continuum limit which is in full agreement with that obtained by analytic continuation.

The paper is organized as follows. In Sec. II, we provide the details regarding the lattice discretization adopted in this study, the various prescriptions to determine $\kappa$ that we have explored and the observables involved in such a prescription. In Sec. III, we illustrate our numerical results and, finally, in Sec. IV, we discuss our conclusions.

\section{NUMERICAL METHODS}

As in our previous studies, Refs. [23,24], we have considered a rooted stout staggered discretization of the $N_{f}=2+1$ QCD partition function:

$$
\begin{gathered}
\mathcal{Z}=\int \mathcal{D} U e^{-\mathcal{S}_{\mathrm{YM}}} \prod_{f=u, d, s} \operatorname{det}\left(M_{\mathrm{st}}^{f}\left[U, \mu_{f, I}\right]\right)^{1 / 4}, \\
\mathcal{S}_{\mathrm{YM}}=-\frac{\beta}{3} \sum_{i, \mu \neq \nu}\left(\frac{5}{6} W_{i ; \mu \nu}^{1 \times 1}-\frac{1}{12} W_{i, \mu \nu}^{1 \times 2}\right), \\
\left(M_{\mathrm{st}}^{f}\right)_{i, j}=\operatorname{am}_{f} \delta_{i, j}+\sum_{\nu=1}^{4} \frac{\eta_{i ; \nu}}{2}\left[e^{a \mu_{f} \delta_{\nu, 4}} U_{i ; \nu}^{(2)} \delta_{i, j-\hat{\nu}}\right. \\
\left.-e^{-a \mu_{f} \delta_{\nu, 4}} U_{i-\hat{\nu} ; \nu}^{(2) \dagger} \delta_{i, j+\hat{\nu}}\right]
\end{gathered}
$$

where $\mathcal{S}_{\mathrm{YM}}$ is the tree level Symanzik improved gauge action $[30,31]$, written in terms of the original link variables through traces of $n \times m$ rectangular loops, $W_{i ; \mu \nu}^{n \times m}$, while the fermion matrix $\left(M_{\mathrm{st}}^{f}\right)_{i, j}$ is built up in terms of the two times stout-smeared [32] links $U_{i ; \nu}^{(2)}$, with an isotropic smearing parameter $\rho=0.15$; finally, the rooting procedure is used to remove the residual fourth degeneracy of staggered fermions (see Ref. [33] for a discussion of possible related systematics.). Note that the quark chemical potentials are treated as external sources, and are set to zero in the simulations.

The quark mass spectrum has been chosen so as to have two degenerate light quarks, $m_{u}=m_{d} \equiv m_{l}$. Standard thermal boundary conditions in the temporal direction have been set for bosonic and fermionic degrees of freedom. The temperature of the system, $T=1 /\left(N_{t} a\right)$, has been changed, for fixed $N_{t}$, by changing the lattice spacing $a$ while staying on a line of constant physics [34,35], corresponding to a pseudo-Goldstone pion mass $m_{\pi} \simeq$ $135 \mathrm{MeV}$ and a strange-to-light mass ratio $m_{s} / m_{l}=28.15$.

\section{A. Physical observables used to locate $T_{c}$ and their renormalization}

As in Refs. [23,24], the determination of the pseudocritical temperature $T_{c}$ will be based on chiral symmetry restoration, which is the leading phenomenon in the presence of light quark masses. In particular, we will consider the light quark condensate,

$$
\langle\bar{\psi} \psi\rangle_{l}=\frac{T}{V} \frac{\partial \log Z}{\partial m_{l}}=\langle\bar{u} u\rangle+\langle\bar{d} d\rangle,
$$

where

$$
\langle\bar{\psi} \psi\rangle_{f}=\frac{T}{V} \frac{\partial \log Z}{\partial m_{f}},
$$

and $V$ is the spatial volume. The light quark condensate is affected by additive and multiplicative renormalizations and, as in Refs. [23,24], we consider two different renormalization prescriptions. The first one is

$$
\langle\bar{\psi} \psi\rangle_{r 1}(T) \equiv \frac{\left[\langle\bar{\psi} \psi\rangle_{l}-\frac{2 m_{l}}{m_{s}}\langle\bar{s} s\rangle\right](T)}{\left[\langle\bar{\psi} \psi\rangle_{l}-\frac{2 m_{l}}{m_{s}}\langle\bar{s} s\rangle\right](T=0)},
$$

and has been introduced in Ref. [36]: the leading additive renormalization, which is linear in the quark mass, cancels in the difference with the strange condensate (even if possible additive logarithmic divergences could still be present), while the multiplicative renormalization, being independent of $\mathrm{T}$, drops out by normalizing with respect to quantities measured at $T=0$ and at the same UV cutoff. The second definition, introduced in Ref. [29], exploits $T=0$ quantities to perform the additive renormalization and the value of the bare quark mass to take care of multiplicative ones:

$$
\langle\bar{\psi} \psi\rangle_{r 2}=\frac{m_{l}}{m_{\pi}^{4}}\left(\langle\bar{\psi} \psi\rangle_{l}-\langle\bar{\psi} \psi\rangle_{l}(T=0)\right) .
$$

The location of $T_{c}$ is usually defined, in terms of the renormalized light condensate, as the point of maximum slope, i.e., the point where $\langle\bar{\psi} \psi\rangle^{r}$ has an inflection point as 
a function of $T$ and the absolute value of $\partial\langle\bar{\psi} \psi\rangle^{r} / \partial T$ reaches a maximum. Alternatively, one can look at the peak of the chiral susceptibility, i.e., the maximum of $\chi_{\bar{\psi} \psi} \equiv$ $\partial\langle\bar{\psi} \psi\rangle^{r} / \partial m_{l}$. Studies exploiting analytic continuation have considered both definitions and then monitored the behavior of $T_{c}$ as a function of the imaginary baryon chemical potential in order to determine $\kappa$. In our case, the determination of $\kappa$ will be based on the matching of derivatives with respect to $T$ and $\mu_{B}$ computed at $\mu_{B}=0$; the main error source will be the statistical one, which is larger and larger as one considers observables representing higherorder derivatives. For this reason, we will limit ourselves to the analysis of the renormalized chiral condensate, which is the lowest derivative, while starting from a second-order derivative like the chiral susceptibility would be much more difficult.

\section{B. Possible definitions of $\boldsymbol{\kappa}$ via Taylor expansion}

The most natural extension to finite $\mu_{B}$ of the prescription to locate $T_{c}$ in terms of $\langle\bar{\psi} \psi\rangle^{r}$ is to still look for an inflection point, i.e., a point where $\partial^{2}\langle\bar{\psi} \psi\rangle^{r} / \partial T^{2}=0$. In order to understand how $T_{c}$ will move, at the lowest order in $\mu_{B}$, following this prescription, we need to consider a Taylor expansion of $\langle\bar{\psi} \psi\rangle^{r}(T)$

$$
\langle\bar{\psi} \psi\rangle^{r}\left(T, \mu_{B}\right)=A(T)+B(T) \mu_{B}^{2}+O\left(\mu_{B}^{4}\right)
$$

where

$$
\begin{aligned}
& A(T) \equiv\langle\bar{\psi} \psi\rangle^{r}(T, 0) \\
& B(T) \equiv \frac{\partial\langle\bar{\psi} \psi\rangle^{r}}{\partial\left(\mu_{B}^{2}\right)}(T, 0) .
\end{aligned}
$$

The prescription is then to require

$$
\begin{aligned}
0 & =\frac{\partial^{2}\langle\bar{\psi} \psi\rangle^{r}}{\partial T^{2}}\left(T, \mu_{B}\right)=A^{\prime \prime}(T)+B^{\prime \prime}(T) \mu_{B}^{2} \\
& =A^{\prime \prime}\left(T_{c}\right)+A^{\prime \prime \prime}\left(T_{c}\right) t+\left(B^{\prime \prime}\left(T_{c}\right)+B^{\prime \prime \prime}\left(T_{c}\right) t\right) \mu_{B}^{2},
\end{aligned}
$$

where the quantities $A^{\prime \prime}, B^{\prime \prime}, A^{\prime \prime \prime}$ and $B^{\prime \prime \prime}$ represent secondand third-order derivatives of $A(T)$ and $B(T)$ with respect to $T, t \equiv T-T_{c}$, and we have performed a lowest-order Taylor expansion around the pseudocritical temperature at $\mu_{B}=0, T_{c}$. Solving Eq. (11) for $t$, one obtains

$$
t=\frac{-B^{\prime \prime}\left(T_{c}\right)}{A^{\prime \prime \prime}\left(T_{c}\right)+B^{\prime \prime \prime}\left(T_{c}\right) \mu_{B}^{2}} \mu_{B}^{2}=-\frac{B^{\prime \prime}\left(T_{c}\right)}{A^{\prime \prime \prime}\left(T_{c}\right)} \mu_{B}^{2}+O\left(\mu_{B}^{4}\right),
$$

and, finally, following the definition of $\kappa$ in Eq. (1), one obtains

$$
\begin{aligned}
\kappa & =\frac{B^{\prime \prime}\left(T_{c}\right)}{A^{\prime \prime \prime}\left(T_{c}\right)} T_{c} \\
& =\frac{\left.\frac{\partial^{2}}{\partial T^{2}}\left(\left.\frac{\partial\langle\bar{\psi} \psi\rangle^{r}\left(T, \mu_{B}\right)}{\partial\left(\mu_{B}^{2}\right)}\right|_{\mu_{B}=0}\right)\right|_{T=T_{c}}}{\left.\frac{\partial^{3}}{\partial T^{3}}\langle\bar{\psi} \psi\rangle^{r}(T, 0)\right|_{T=T_{c}}} T_{c} .
\end{aligned}
$$

From a practical point of view, Eq. (13) means that one needs to evaluate both the renormalized condensate and its $\mu_{B}^{2}$ derivative as a function of $T$ around $T_{c}$, and that must be done with enough precision so that, after a suitable interpolation, one is able to compute numerically their third- and second-order derivatives with respect to $T$ at $T_{c}$.

As we shall see, the program above has to face the low statistical accuracy attainable with reasonable statistics, in particular when evaluating the $\mu_{B}^{2}$-derivative and especially on the lattices with higher values of $N_{t}$, which are necessary to take the continuum extrapolation. For this reason, alternative prescriptions for $\kappa$ have been adopted in the literature. For instance, in Ref. [29], the pseudocritical temperature at finite $\mu_{B}$ is defined as the temperature where the renormalized condensate attains the same value as at $T_{c}$ for $\mu_{B}=0$, i.e.:

$$
\left.\langle\bar{\psi} \psi\rangle^{r}\left(T, \mu_{B}^{2}\right)\right|_{T=T_{c}\left(\mu_{B}^{2}\right)} \equiv\langle\bar{\psi} \psi\rangle^{r}\left(T_{c}, 0\right) .
$$

Then, by definition, the differential $d\langle\bar{\psi} \psi \psi\rangle$ must vanish along the curve $T_{c}\left(\mu_{B}\right)$,

$$
d\langle\bar{\psi} \psi\rangle^{r}=\left.\frac{\partial\langle\bar{\psi} \psi\rangle^{r}}{\partial T}\right|_{\mu_{B}=0} d T+\left.\frac{\partial\langle\bar{\psi} \psi\rangle^{r}}{\partial\left(\mu_{B}^{2}\right)}\right|_{\mu_{B}=0} d\left(\mu_{B}^{2}\right)=0,
$$

so that one obtains

$$
\kappa=-T_{c} \frac{d T_{c}}{d\left(\mu_{B}^{2}\right)}=T_{c} \frac{\left.\frac{\partial\langle\bar{\psi} \psi\rangle^{r}}{\partial\left(\mu_{B}^{2}\right)}\right|_{\mu_{B}=0, T=T_{c}}}{\left.\frac{\partial\langle\bar{\psi} \psi\rangle^{r}}{\partial T}\right|_{\mu_{B}=0, T=T_{c}}} .
$$

The advantage of the expression in Eq. (16) with respect to that in Eq. (13) is twofold: one needs to estimate just the first derivative of the renormalized condensate at $T_{c}$, which is more precise and stable against the choice of the interpolating function than the third one, and one does not need to know the dependence of $\partial\langle\bar{\psi} \psi \psi\rangle^{r} / \partial\left(\mu_{B}^{2}\right)$ on $T$, but just its value at $T_{c}$. However, the prescription is debatable, since there is no strict reason that the condensate should stay constant in value at $T_{c}$. However, numerical studies at imaginary chemical potential [23] have shown that it gives results for $T_{c}$ which are compatible, within errors, with those obtained by looking at the inflection point.

In the following we will consider both definitions, and refer to them as $\kappa_{1}$, Eq. (16), and $\kappa_{2}$, Eq. (13). As we shall see, a detailed comparison between the two definitions will 
be possible only on $N_{t}=6$ lattices, where they will give compatible results, while on lattices with larger $N_{t}$ the statistical errors attained for $\kappa_{2}$ will make it practically useless, so that our present continuum extrapolation will be based on $\kappa_{1}$ alone. Yet, $\kappa_{1}$ is exactly the prescription adopted in Ref. [29], so that a strict comparison will be possible with the results reported there.

Notice that other prescriptions can be found in the literature, which will not be explored in this study. For instance, the determination reported in Ref. [28] (see also Refs. [27,37,38]) assumes a behavior for the pseudocritical temperature which is dictated by the critical scaling around the possible second-order point in the $O(4)$ universality class located at $m_{l}=0$.

\section{Observables needed to determine $\kappa$ and setup of chemical potentials}

Apart from the renormalized chiral condensate, which has been already defined above, the other quantity needed for our study is its derivative with respect to $\mu_{B}^{2}$. Looking at Eqs. (7) and (8), one realizes that such a derivative is trivially obtained combining the derivatives of the finite temperature flavor condensates with respect to $\mu_{B}^{2}$, since zero temperature quantities are independent of $\mu_{B}$ around $\mu_{B}=0$. Therefore, we need to compute

$$
\begin{aligned}
\left.\frac{\partial\langle\bar{\psi} \psi\rangle_{f}}{\partial\left(\mu_{B}^{2}\right)}\right|_{\mu_{B}=0}= & \left.\frac{1}{T^{2}} \frac{\partial\langle\bar{\psi} \psi\rangle_{f}}{\partial\left(\left(\frac{\mu_{B}}{T}\right)^{2}\right)}\right|_{\mu_{B}=0} \\
= & \left.\frac{1}{T^{2}} \frac{1}{2} \frac{\partial^{2}\langle\bar{\psi} \psi\rangle_{f}}{\partial\left(\frac{\mu_{B}}{T}\right)^{2}}\right|_{\mu_{B}=0} \\
= & \frac{1}{T^{2}} \frac{1}{2}\left(\left\langle\left(n^{2}+n^{\prime}\right) \bar{\psi} \psi_{f}\right\rangle-\left\langle n^{2}+n^{\prime}\right\rangle\left\langle\bar{\psi} \psi_{f}\right\rangle\right. \\
& \left.+\left\langle 2 n \bar{\psi} \psi_{f}^{\prime}+\bar{\psi} \psi_{f}^{\prime \prime}\right\rangle\right),
\end{aligned}
$$

where the relevant operators entering previous expression are defined as

$$
\begin{aligned}
\bar{\psi} \psi_{f} & =\frac{T}{V} \frac{1}{4} \operatorname{Tr}\left[M_{f}^{-1}\right], \\
\bar{\psi} \psi_{f}^{\prime} & =\frac{T}{V} \frac{1}{4} \operatorname{Tr}\left[-M_{f}^{-1} M_{f}^{\prime} M_{f}^{-1}\right], \\
\bar{\psi} \psi_{f}^{\prime \prime} & =\frac{T}{V} \frac{1}{4} \operatorname{Tr}\left[2 M_{f}^{-1} M_{f}^{\prime} M_{f}^{-1} M_{f}^{\prime} M_{f}^{-1}-M_{f}^{-1} M_{f}^{\prime \prime} M_{f}^{-1}\right], \\
n & =\sum_{f=u d s} \frac{1}{4} \operatorname{Tr}\left[M_{f}^{-1} M_{f}^{\prime}\right], \\
n^{\prime} & =\sum_{f=u d s} \frac{1}{4} \operatorname{Tr}\left[M_{f}^{-1} M_{f}^{\prime \prime}-M_{f}^{-1} M_{f}^{\prime} M_{f}^{-1} M_{f}^{\prime}\right],
\end{aligned}
$$

while $M_{f}^{\prime}$ and $M_{f}^{\prime \prime}$ represent first and second derivatives of the fermion matrix, defined in Eq. (4), with respect to $\mu_{B}$, computed at $\mu_{B}=0$.
The way in which such derivatives, $M_{f}^{\prime}$ and $M_{f}^{\prime \prime}$, are actually taken depends on the quark flavor $f$ and specifies our setup of quark chemical potentials. In particular, as in Refs. [23,24,29], we set $\mu_{u}=\mu_{d}=\mu_{l}=\mu_{B} / 3$ and $\mu_{s}=0$. Therefore, for the strange flavor, we have $M_{s}^{\prime}=M_{s}^{\prime \prime}=0$, so that $\bar{\psi} \psi_{s}^{\prime}$ and $\bar{\psi} \psi_{s}^{\prime \prime}$ trivially vanish. Instead, for $f=u, d$, considering Eq. (4) and taking into account that $\partial / \partial \mu_{B}=(1 / 3) \partial / \partial \mu_{l}$, we have

$$
\begin{aligned}
& M_{f_{i, j}}^{\prime}=\frac{\eta_{i ; 4}}{6}\left[U_{i ; 4}^{(2)} \delta_{i, j-\hat{4}}+U_{i-\hat{4} ; 4}^{(2) \dagger} \delta_{i, j+\hat{4}}\right], \\
& M_{f_{i, j}}^{\prime \prime}=\frac{\eta_{i ; 4}}{18}\left[U_{i ; 4}^{(2)} \delta_{i, j-\hat{4}}-U_{i-\hat{4} ; 4}^{(2) \dagger} \delta_{i, j+\hat{4}}\right] .
\end{aligned}
$$

All traces appearing in Eq. (18) have been computed, as usual, by multiple noisy estimators, paying attention not to combine the same random vectors when estimating the product of traces in order to avoid cross-correlations.

\section{NUMERICAL RESULTS}

We performed numerical simulations on four different lattices, with dimensions $16^{3} \times 6,24^{3} \times 6,32^{3} \times 8$ and $40^{3} \times 10$, and adopting the bare parameters reported in Table I in order to stay on a line of constant physics. The scale determination is affected by an overall systematic error of the order of 2\%-3\% [34,35]; however, this is not relevant to our final results, which are based on the determination of dimensionless ratios of quantities measured at the critical temperature. We have adopted the standard rational hybrid Monte Carlo algorithm [39-41] implemented in two different codes, one running on standard clusters (NISSA), the other on GPUs (OpenStaPLE $[42,43])$ and developed in OpenACC starting from previous GPU implementations [44].

A total of $\approx 20-30 K$ and $\approx 6 K$ molecular dynamics trajectories have been generated, for each value of $\beta$, respectively, on the two coarsest lattices and on the $N_{t}=8$ lattice. On the $N_{t}=10$ lattice, the derivative of the chiral condensate has been measured exploiting a dedicated highstatistics simulation performed at the critical temperature and consisting of $\approx 100 K$ trajectories, while for the values of the chiral condensate around $T_{c}$ we have relied on results obtained in Ref. [24]. Also for the values of the chiral condensate at zero temperature, which are needed to obtain renormalized quantities, we have used results obtained in previous studies [23,24]. Finally, all traces needed in our computations [see Eq. (18)] have been estimated, respectively, for the $N_{t}=6,8,10$ lattices, every 10,20 and 50 trajectories by means of noisy estimators, adopting 256, 512 and 512 random vectors for each flavor and for each configuration.

Numerical results obtained on $N_{t}=6,8$ for the chiral condensate and for its derivative with respect to $\mu_{B}^{2}$ are reported, for both renormalization procedures, respectively, 
TABLE I. List of the bare quark masses and lattice spacings used in our simulations. Bare parameters have been chosen so as to stay on a line of constant physics with a physical value of the pseudo-Goldstone pion mass, interpolating results reported in Refs. [34,35]. The strange-to-light mass ratio has been fixed to $m_{s} / m_{l}=28.15$.

\begin{tabular}{llll}
\hline \hline$\beta$ & \multicolumn{1}{c}{$m_{s}$} & $a(f m)$ & Lattice \\
\hline 3.49 & 0.132 & 0.2556 & $N_{t}=6$ \\
3.51 & 0.121 & 0.2425 & $N_{t}=6$ \\
3.52 & 0.116 & 0.2361 & $N_{t}=6$ \\
3.525 & 0.11350 & 0.23297 & $N_{t}=6$ \\
3.53 & 0.111 & 0.2297 & $N_{t}=6$ \\
3.535 & 0.10873 & 0.22663 & $N_{t}=6$ \\
3.54 & 0.10643 & 0.2235 & $N_{t}=6$ \\
3.545 & 0.10419 & 0.22039 & $N_{t}=6$ \\
3.55 & 0.10200 & 0.2173 & $N_{t}=6$ \\
3.555 & 0.099864 & 0.21424 & $N_{t}=6$ \\
3.56 & 0.09779 & 0.2112 & $N_{t}=6$ \\
3.565 & 0.095750 & 0.20820 & $N_{t}=6$ \\
3.57 & 0.09378 & 0.2052 & $N_{t}=6$ \\
3.58 & 0.08998 & 0.1994 & $N_{t}=6$ \\
3.60 & 0.08296 & 0.1881 & $N_{t}=6,8$ \\
3.62 & 0.07668 & 0.1773 & $N_{t}=8$ \\
3.63 & 0.07381 & 0.1722 & $N_{t}=8$ \\
3.635 & 0.07240 & 0.1697 & $N_{t}=8$ \\
3.64 & 0.07110 & 0.1672 & $N_{t}=8$ \\
3.645 & 0.06978 & 0.1648 & $N_{t}=8$ \\
3.655 & 0.06731 & 0.1601 & $N_{t}=8$ \\
3.66 & 0.06615 & 0.1579 & $N_{t}=8$ \\
3.665 & 0.06500 & 0.1557 & $N_{t}=8$ \\
3.67 & 0.06390 & 0.1535 & $N_{t}=8$ \\
3.675 & 0.06284 & 0.1514 & $N_{t}=8$ \\
3.68 & 0.06179 & 0.1493 & $N_{t}=8$ \\
3.69 & 0.05982 & 0.1453 & $N_{t}=8$ \\
3.71 & 0.05624 & 0.1379 & $N_{t}=8$ \\
3.74 & 0.05168 & 0.1280 & $N_{t}=10$ \\
\hline \hline & & & \\
\hline \hline
\end{tabular}

in Figs. 1-4. As for the derivative, we report in all cases the dimensionless combination $T_{c}^{2} B=T_{c}^{2} \partial\langle\bar{\psi} \psi\rangle_{r} / \partial\left(\mu_{B}^{2}\right)$. Statistical errors have been estimated by a jackknife analysis.

Reported results have been fitted in order to obtain the quantities $A^{\prime}, A^{\prime \prime \prime}, B$ and $B^{\prime \prime}$ computed at the pseudocritical temperature needed to determine $\kappa$, where derivatives are taken with respect to the temperature [see Eqs. (13) and (16)]:

$$
\kappa_{1}=\frac{1}{T_{c}} \frac{T_{c}^{2} B}{A^{\prime}} ; \quad \kappa_{2}=\frac{1}{T_{c}} \frac{T_{c}^{2} B^{\prime \prime}}{A^{\prime \prime \prime}}
$$

In order to estimate the systematic uncertainty related to the choice of the fitting function, we have tried different Ansätze which are summarized in Table II. In particular, for the renormalized condensate we have adopted an arctangent, $A=P_{1}+P_{2} \operatorname{atan}\left(P_{3}\left(T-T_{c}\right)\right)$, a hyperbolic tangent and a cubic polynomial, while for its $\mu_{B}^{2}$ derivative we have
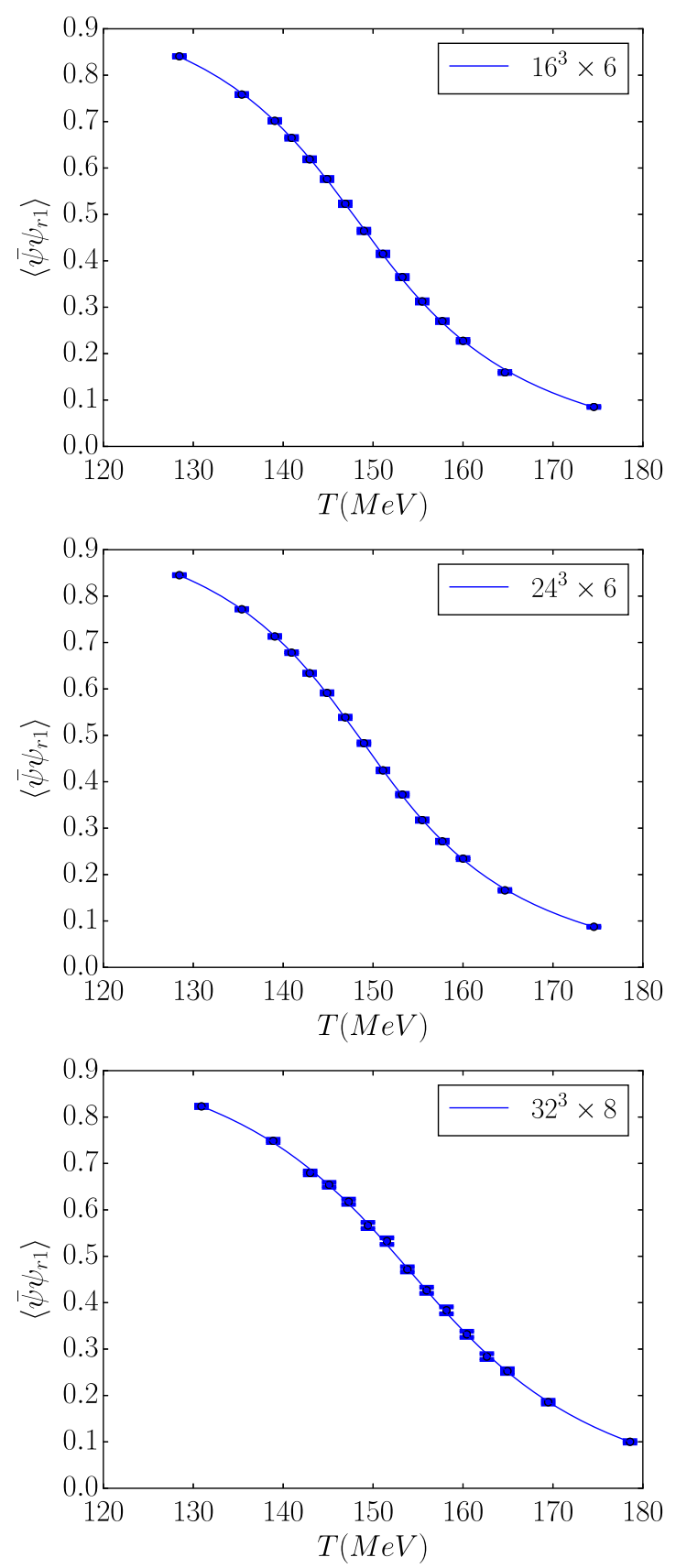

FIG. 1. Renormalized chiral condensate $\left\langle\bar{\psi} \psi_{r 1}\right\rangle$ for $N_{t}=6$ and $N_{t}=8$ lattices.

considered a Lorentzian function $T_{c}^{2} B=\frac{P_{1}}{P_{2}^{2}+\left(T-P_{3}\right)^{2}}$, a parabola and a cubic spline. All best-fit results for $A^{\prime}$, $A^{\prime \prime \prime}, T_{c}^{2} B$ and $T_{c}^{2} B^{\prime \prime}$ evaluated at $T_{c}$ are reported in Tables III and IV: reported errors include the systematic one related to the choice of the fit range. Results obtained for the pseudocritical temperature $T_{c}$ are instead reported in Table V.

In principle, the uncertainty in the determination of $T_{c}$ should contribute to the error given for $A^{\prime}, A^{\prime \prime \prime}, B$ and $B^{\prime \prime}$ computed at $T_{c}$; however, this contribution turns out to be 

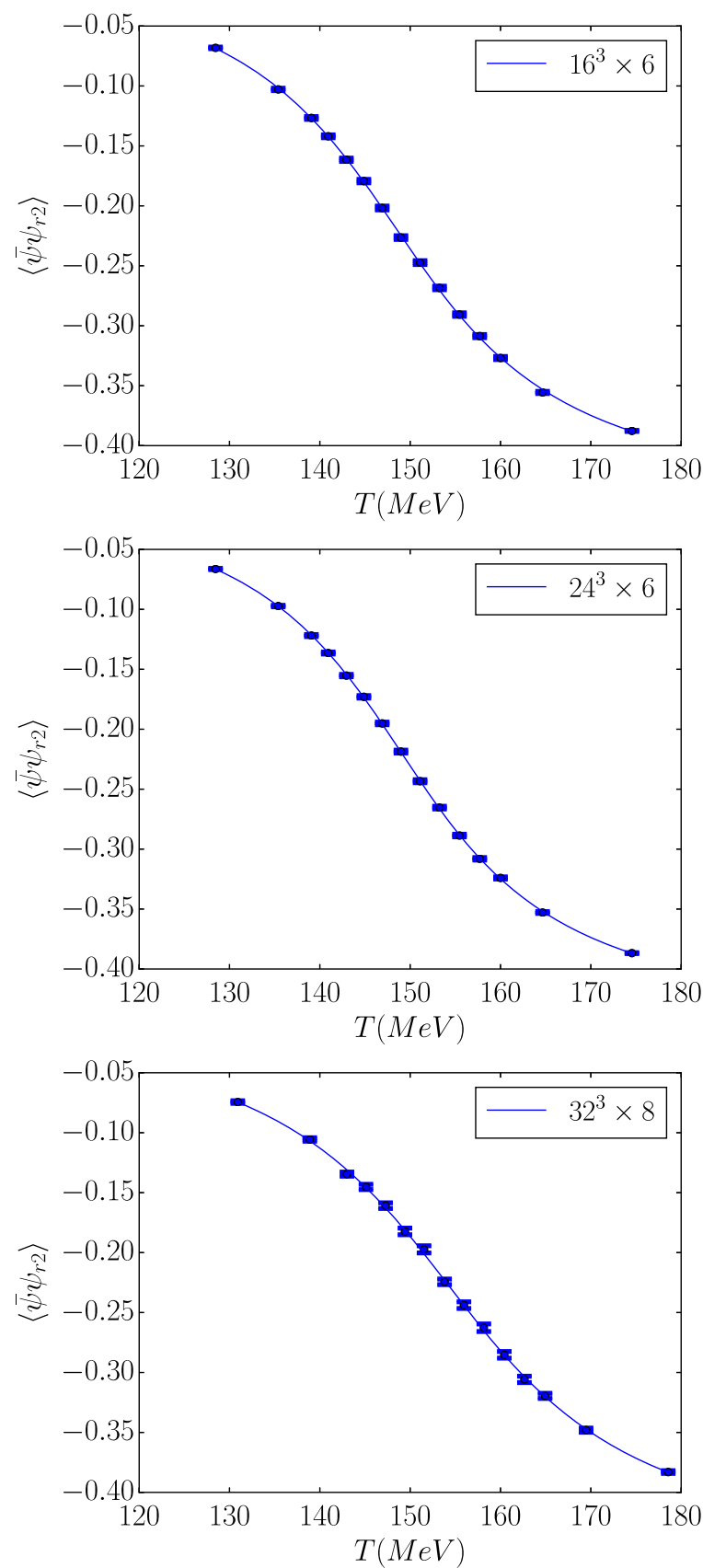

FIG. 2. Renormalized chiral condensate $\left\langle\bar{\psi} \psi_{r 2}\right\rangle$ for $N_{t}=6$ and $N_{t}=8$ lattices.

negligible in most cases, apart from the $40^{3} \times 10$ (and in particular for $B$ ) where it is marginally appreciable because of the larger uncertainty on $T_{c}$. For the determinations of $T_{c}$ and $A^{\prime}$ on the $N_{t}=10$ lattice, we reused the data already obtained in Ref. [24], while $B$ has been obtained from the single dedicated simulation performed at $T \simeq T_{c}$. Results for $A^{\prime}$ and $B$ are reported. respectively, in Tables VI and VII; the estimate for the error on $B\left(T_{c}\right)$ stemming from the uncertainty on $T_{c}$ has been based on the data available for $B$ as a function of $T$ on the $24^{3} \times 6$ lattice.
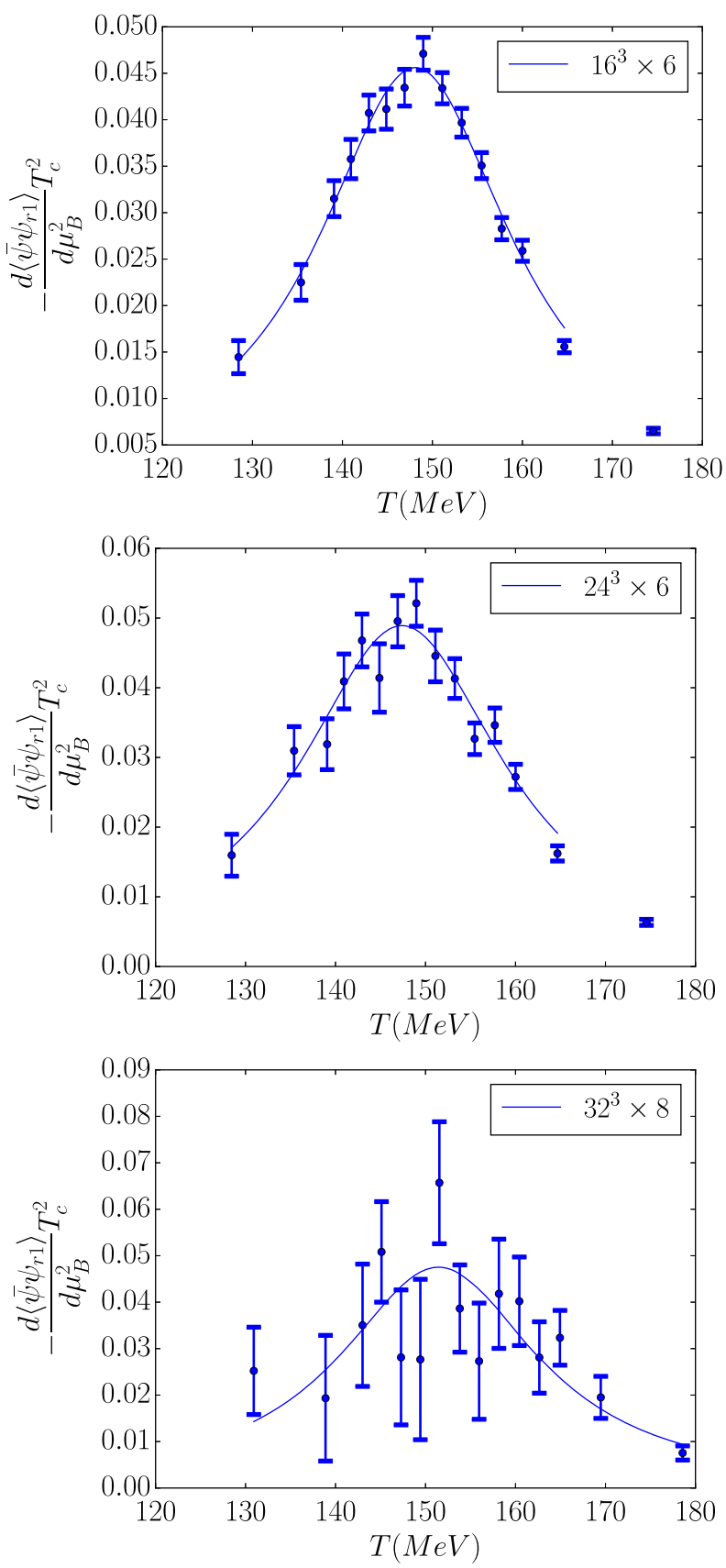

FIG. 3. First derivative with respect to $\mu_{B}^{2}$ of $\left\langle\bar{\psi} \psi_{r 1}\right\rangle$ for $N_{t}=6$ and $N_{t}=8$ lattices.

As it can be appreciated from Tables III and IV, the systematic uncertainties related to the choice of the fitting function are in a few cases comparable or larger than statistical errors. For this reason, in order to obtain our final estimates for $\kappa_{1}$ and $\kappa_{2}$, which are based on Eq. (21) and are reported in Table VIII, we have considered the dispersion of values corresponding to all possible combinations of different fitting functions, and added it, when appreciable, to the statistical error. As one can see, present statistics are not enough to reach reliable estimates of $B^{\prime \prime}$ on lattices with 

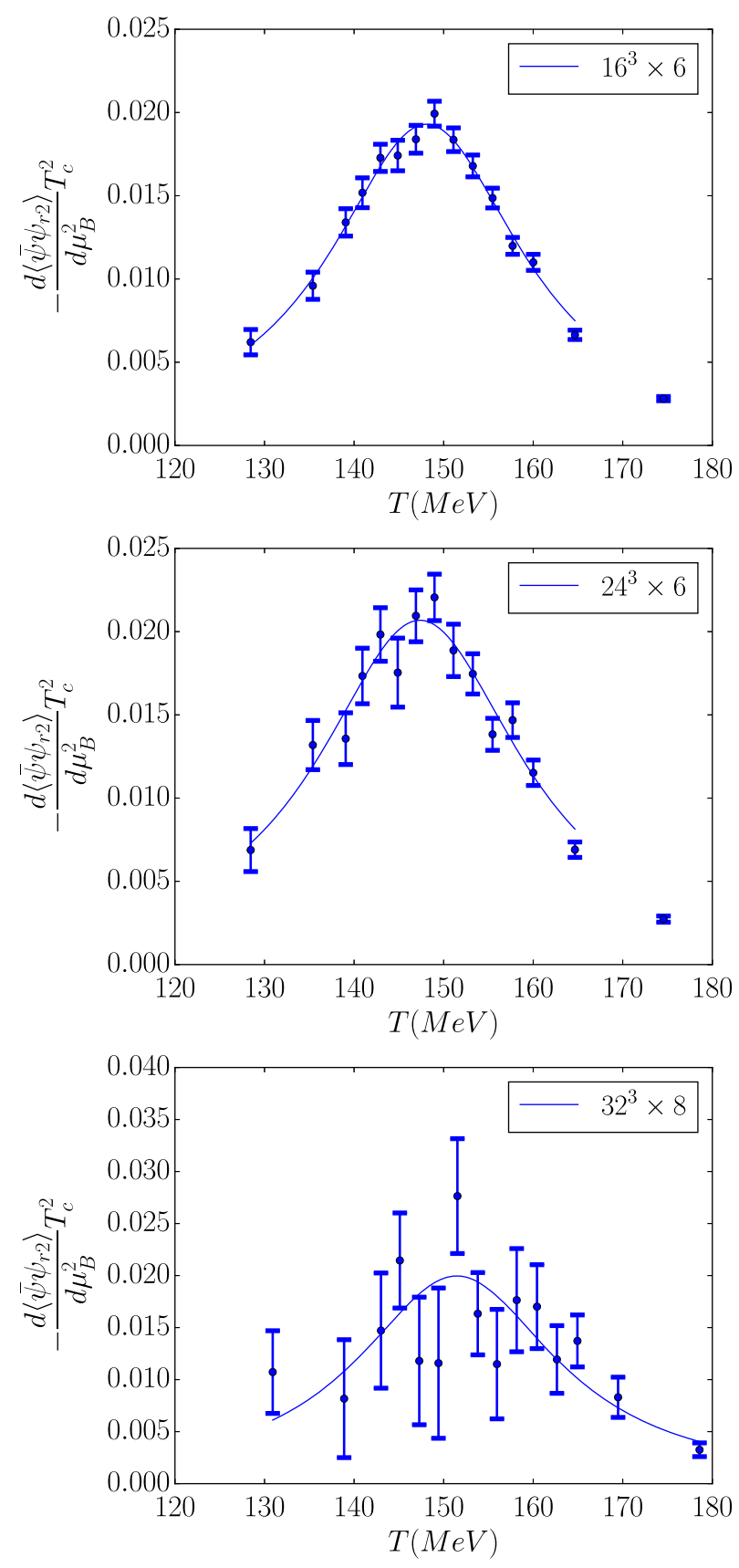

FIG. 4. First derivative with respect to $\mu_{B}^{2}$ of $\left\langle\bar{\psi} \psi_{r 2}\right\rangle$ for $N_{t}=6$ and $N_{t}=8$ lattices.

TABLE II. Summary of the symbols used in the following tables to describe the Ansatz for the fitting function.

\begin{tabular}{cc}
\hline \hline Symbol & Fit function \\
\hline A & Atan \\
T & Tanh \\
C & Cubic \\
L & Lorentzian \\
P & Parabola \\
S & Spline \\
\hline \hline
\end{tabular}

TABLE III. Values obtained for $A^{\prime}\left(T_{c}\right)$ and $A^{\prime \prime \prime}\left(T_{c}\right)$ on lattices with $N_{t}=6$ and 8. Indexes $r 1$ and $r 2$ refer to the two different definitions of the renormalized condensate. Derivatives have been take with respect to the physical temperature, therefore they are reported in $\mathrm{MeV}^{-1}$ and $\mathrm{MeV}^{-3}$ units, respectively, for $A^{\prime}$ and $A^{\prime \prime \prime}$.

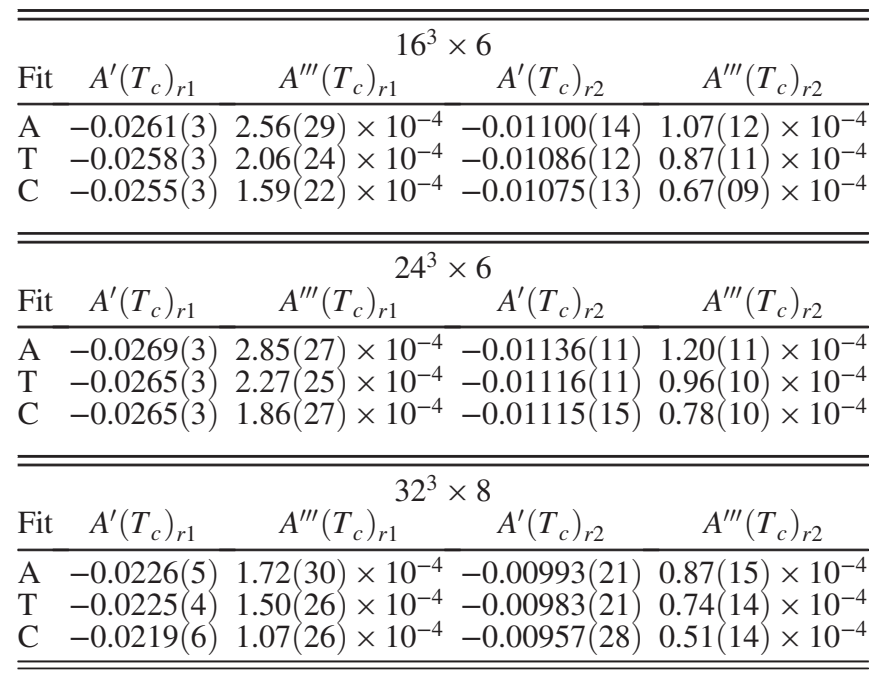

$N_{t}>6$, where they are affected by errors of the order of $50 \%$.

\section{A. Discussion of results on $N_{t}=6$ lattices: Finite size effects and comparison between $\kappa_{1}$ and $\kappa_{2}$}

Results obtained on $N_{t}=6$ for different spatial sizes permit us to make an assessment of the relevance of finite

TABLE IV. Values obtained for $T_{c}^{2} B\left(T_{c}\right)$ and $T_{c}^{2} B^{\prime \prime}\left(T_{c}\right)$ on lattices with $N_{t}=6$ and 8. Indexes $r 1$ and $r 2$ refer to the two different definitions of the renormalized condensate. $T_{c}^{2} B\left(T_{c}\right)$ is dimensionless while $T_{c}^{2} B^{\prime \prime}\left(T_{c}\right)$ is given in $\mathrm{MeV}^{-2}$ units.

\begin{tabular}{lcccc}
\hline \hline \multicolumn{5}{c}{$16^{3} \times 6$} \\
Fit & $T_{c}^{2} B\left(T_{c}\right)_{r 1}$ & $T_{c}^{2} B^{\prime \prime}\left(T_{c}\right)_{r 1}$ & $T_{c}^{2} B\left(T_{c}\right)_{r 2}$ & $T_{c}^{2} B^{\prime \prime}\left(T_{c}\right)_{r 2}$ \\
\hline $\mathrm{L}$ & $0.0461(9)$ & $-5.8(7) \times 10^{-4}$ & $0.0195(4)$ & $-2.50(29) \times 10^{-4}$ \\
$\mathrm{P}$ & $0.0448(9)$ & $-3.5(5) \times 10^{-4}$ & $0.0189(4)$ & $-1.46(21) \times 10^{-4}$ \\
$\mathrm{~S}$ & $0.0455(9)$ & $-4.7(4) \times 10^{-4}$ & $0.0192(4)$ & $-1.97(16) \times 10^{-4}$ \\
\hline \hline & \multicolumn{5}{c}{$24^{3} \times 6$} \\
Fit & $T_{c}^{2} B\left(T_{c}\right)_{r 1}$ & $T_{c}^{2} B^{\prime \prime}\left(T_{c}\right)_{r 1}$ & $T_{c}^{2} B\left(T_{c}\right)_{r 2}$ & $T_{c}^{2} B^{\prime \prime}\left(T_{c}\right)_{r 2}$ \\
\hline $\mathrm{L}$ & $0.0487(16)$ & $-5.0(8) \times 10^{-4}$ & $0.0206(7)$ & $-2.1(3) \times 10^{-4}$ \\
$\mathrm{P}$ & $0.0479(17)$ & $-3.9(8) \times 10^{-4}$ & $0.0202(7)$ & $-1.7(4) \times 10^{-4}$ \\
$\mathrm{~S}$ & $0.0485(17)$ & $-4.9(8) \times 10^{-4}$ & $0.0205(7)$ & $-2.0(3) \times 10^{-4}$ \\
\hline \hline & \multicolumn{5}{c}{$32^{3} \times 8$} \\
Fit & $T_{c}^{2} B\left(T_{c}\right)_{r 1}$ & $T_{c}^{2} B^{\prime \prime}\left(T_{c}\right)_{r 1}$ & $T_{c}^{2} B\left(T_{c}\right)_{r 2}$ & $T_{c}^{2} B^{\prime \prime}\left(T_{c}\right)_{r 2}$ \\
\hline $\mathrm{L}$ & $0.044(5)$ & $-3.2(1.8) \times 10^{-4}$ & $0.0187(21)$ & $-1.3(7) \times 10^{-4}$ \\
$\mathrm{P}$ & $0.041(4)$ & $-1.6(0.9) \times 10^{-4}$ & $0.0172(18)$ & $-0.7(4) \times 10^{-4}$ \\
$\mathrm{~S}$ & $0.042(6)$ & $-2.9(2.1) \times 10^{-4}$ & $0.0175(25)$ & $-1.2(9) \times 10^{-4}$ \\
\hline \hline
\end{tabular}


TABLE V. Values obtained for the critical temperature from the fits. Reported errors take into account the systematic uncertainty related to the choice of the fitting function and range, but not the overall uncertainty on the determination of the physical scale, which is of the order of $2 \%-3 \%$ [34,35]. Values reported for the $40^{3} \times 10$ lattice are based on results reported in Ref. [24].

\begin{tabular}{lll}
\hline \hline Lattice & $T_{c}\left(\bar{\psi} \psi_{r 1}\right)$ & $T_{c}\left(\bar{\psi} \psi_{r 2}\right)$ \\
\hline $16^{3} \times 6$ & $148.5(3)$ & $148.6(3)$ \\
$24^{3} \times 6$ & $149.1(2)$ & $149.2(2)$ \\
$32^{3} \times 8$ & $154.4(4)$ & $154.7(4)$ \\
$40^{3} \times 10$ & $154.7(1.6)$ & $154.4(1.6)$ \\
\hline \hline
\end{tabular}

TABLE VI. Values obtained for $A^{\prime}\left(T_{c}\right)$ on the $40^{3} \times 10$ lattice, based on results obtained in Ref. [24]. Units and conventions are as in Table III.

\begin{tabular}{lcc}
\hline \hline Fit & $A^{\prime}\left(T_{c}\right)_{r 1}$ & $A^{\prime}\left(T_{c}\right)_{r 2}$ \\
\hline A & $-0.0231(09)$ & $-0.0093(4)$ \\
$\mathrm{T}$ & $-0.0226(11)$ & $-0.0091(4)$ \\
$\mathrm{C}$ & $-0.0215(12)$ & $-0.0089(5)$ \\
\hline \hline
\end{tabular}

TABLE VII. Values obtained for $T_{c}^{2} B\left(T_{c}\right)$ on the $40^{3} \times 10$ lattice. The second error refers to the uncertainty in the determination of the pseudocritical temperature at $\mu_{B}=0$.

\begin{tabular}{lc}
\hline \hline$T_{c}^{2} B\left(T_{c}\right)_{r 1}$ & $T_{c}^{2} B\left(T_{c}\right)_{r 2}$ \\
\hline $0.052(6)(2)$ & $0.0217(25)(10)$ \\
\hline \hline
\end{tabular}

size effects. For a closer comparison, in Fig. 5 we plot together results obtained for $T_{c}^{2} B$ on the different volumes. A mild dependence on the spatial volume is visible for some quantities, like for instance the pseudocritical temperature. However, results obtained for $\kappa$ on the $16^{3} \times 6$ and $24^{3} \times 6$ lattices are in perfect agreement within errors, thus confirming the small sensitivity of $\kappa$ to finite size effects already observed in studies exploiting analytic continuation [23].

Regarding the comparison between $\kappa_{1}$ and $\kappa_{2}$, one observes a slight tendency for $\kappa_{2}$ to be larger than $\kappa_{1}$; however, this is not significant within errors, which are of the order of $30 \%$ for $\kappa_{2}$; therefore, the two determinations

TABLE VIII. Curvature coefficient $\kappa$ obtained for different definitions and lattice sizes.

\begin{tabular}{llclc}
\hline \hline Lattice & $\kappa_{1}\left(\bar{\psi} \psi_{r 1}\right)$ & $\kappa_{2}\left(\bar{\psi} \psi_{r 1}\right)$ & $\kappa_{1}\left(\bar{\psi} \psi_{r 2}\right)$ & $\kappa_{2}\left(\bar{\psi} \psi_{r 2}\right)$ \\
\hline $16^{3} \times 6$ & $0.0119(4)$ & $0.016(5)$ & $0.0119(4)$ & $0.016(5)$ \\
$24^{3} \times 6$ & $0.0122(4)$ & $0.015(4)$ & $0.0122(4)$ & $0.015(4)$ \\
$32^{3} \times 8$ & $0.0126(14)$ & $0.014(9)$ & $0.0121(13)$ & $0.012(8)$ \\
$40^{3} \times 10$ & $0.0146(19)$ & $\cdots$ & $0.0154(21)$ & $\cdots$ \\
\hline \hline
\end{tabular}
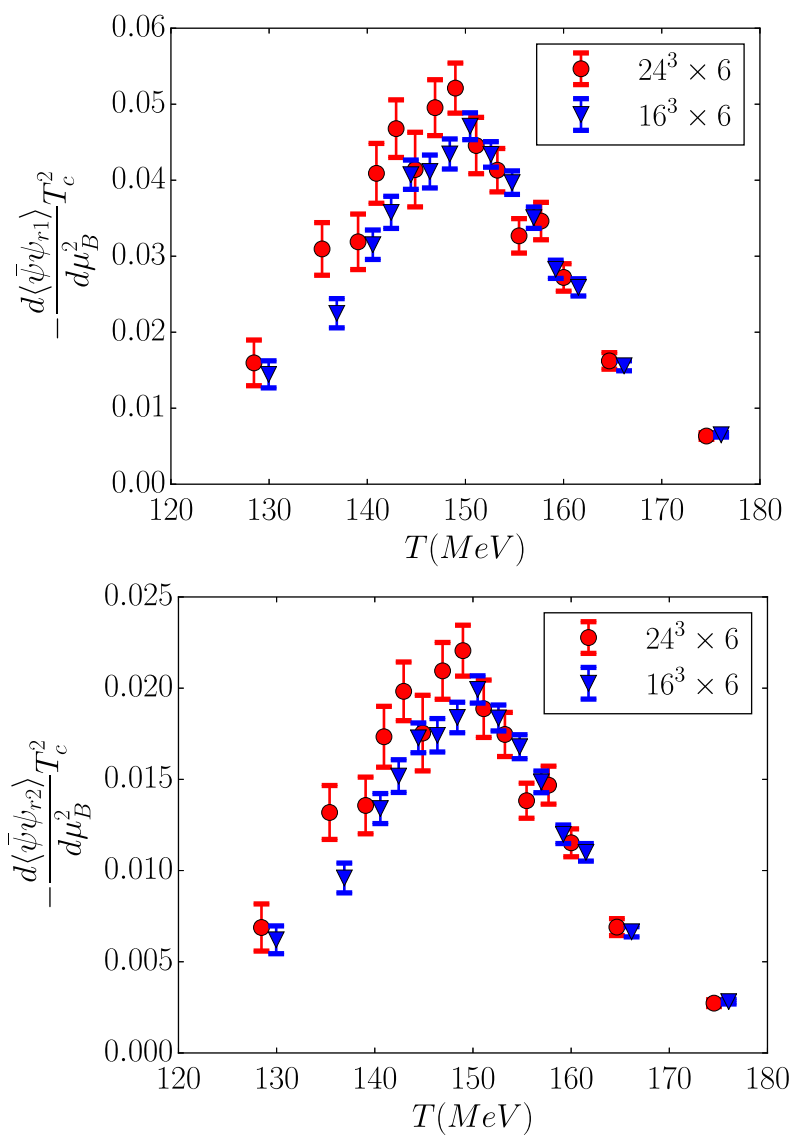

FIG. 5. First derivative with respect to $\mu_{B}^{2}$ of the renormalized condensate: comparison between the results obtained on the $16^{3}$ and the $24^{3}$ lattices for the two different definitions. Data points have been slightly shifted horizontally to improve readability.

are compatible within our present level of accuracy. Finally, no significant difference is observed between determinations obtained with the two different renormalization prescriptions for the chiral condensate.

\section{B. Continuum extrapolation of $\kappa_{1}$ and comparison with analytic continuation}

A continuum extrapolation is presently possible only for $\kappa_{1}$, for which results are available for three different lattice spacings, corresponding to $N_{t}=6,8$ and 10 , while for $\kappa_{2}$ we can just say that no appreciable variations are observable going from $N_{t}=6$ to $N_{t}=8$; however, errors for $N_{t}=8$ are too large to make this statement of any significance. Assuming corrections linear in $a^{2}, \kappa_{1}\left(a^{2}\right)=\kappa_{1}^{\text {cont }}+$ $O\left(a^{2}\right)$, and since $T=\frac{1}{N_{t} a}$, an extrapolation to the continuum limit for $\kappa_{1}$ can be obtained by a best fit of the function

$$
\kappa\left(N_{t}\right)=\kappa^{\mathrm{cont}}+A \frac{1}{N_{t}^{2}} .
$$

The values of $\kappa_{1}$ obtained on the $24^{3} \times 6,32^{3} \times 8$ and $40^{3} \times 10$ lattices are reported in Fig. 6, where are 


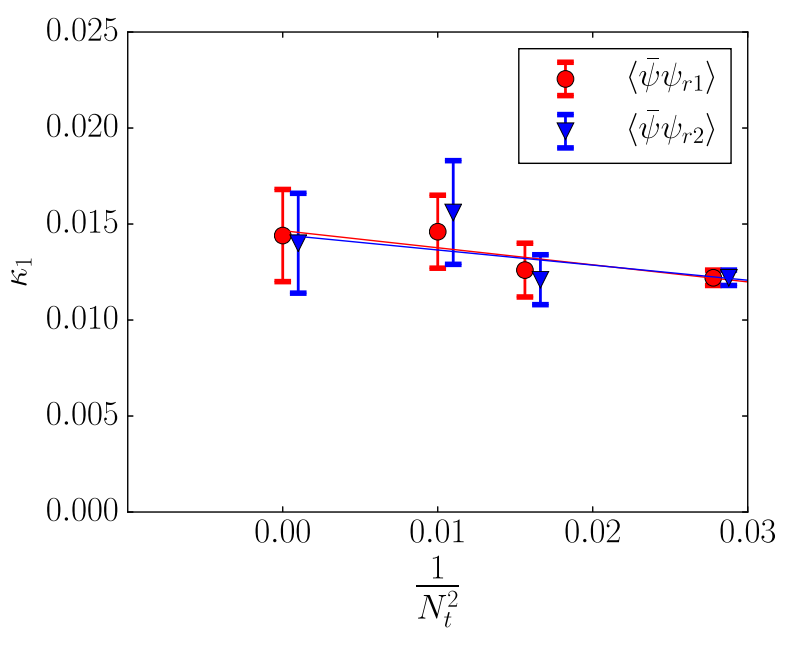

FIG. 6. Values obtained for $\kappa_{1}$ on different values of $N_{t}$ and continuum extrapolation.

also illustrated the results of the continuum extrapolation, which gives back $\kappa_{1}^{\text {cont }}\left(\bar{\psi} \psi_{r 1}\right)=0.0147(22)$ and $\kappa_{1}^{\text {cont }}\left(\bar{\psi} \psi_{r 2}\right)=0.0144(26)$, with a reduced chi-squared, respectively, 0.42 and 1.38 .

As a final estimate, we quote the average value $\kappa_{1}=0.0145(25)$, which is in very good agreement within errors with previous results obtained via analytic continuation, in particular $\kappa=0.0135$ (20) from Ref. [24] where the same discretization and numerical setup of chemical potentials have been adopted.

Apart from the continuum limit, a direct comparison with analytic continuation can be made separately for the different lattice sizes and for the different definitions of $\kappa$. This is possible in particular exploiting the results reported in Ref. [23], where values of both $\kappa_{1}$ and $\kappa_{2}$ have been reported for $16^{3} \times 6,24^{3} \times 6$ and $32^{3} \times 8$ lattices and for both renormalizations of the chiral condensate. An inspection of Table IX, where results from this work and from Ref. $[23]^{1}$ are reported together for lattices where both are available, reveals some tension (at the level of three standard deviations) between Taylor expansion and analytic continuation on the coarsest lattices and for $\kappa_{1}$, which tends to disappear on the finer lattices, also because of the larger statistical errors.

It is interesting that even the tension observed on $N_{t}=6$ lattices for $\kappa_{1}$ disappears as soon as one can correctly estimate systematic errors connected to the truncation of the fitting polynomial in analytic continuation: just for $N_{t}=6$ that was not possible in Ref. [23], because of the limited amount of chemical potentials available for that

\footnotetext{
${ }^{1}$ Notice that the result reported from Ref. [23] for $\kappa_{1}\left(\bar{\psi} \psi_{r 2}\right)$ on the $24^{3} \times 6$ is different from what actually appearing in Table IX of Ref. [23], where 0.0147(3) is reported, instead of 0.0140(3), because of a typo. The number reported here has been checked by fitting directly the pseudocritical temperatures appearing in Table VI of Ref. [23].
}

TABLE IX. Comparison of results obtained for different definitions of $\kappa$ in this work with those reported in Ref. [23] via analytic continuation.

\begin{tabular}{llllll}
\hline \hline Work & Lattice & $\kappa_{1}\left(\bar{\psi} \psi_{r 1}\right)$ & $\kappa_{2}\left(\bar{\psi} \psi_{r 1}\right)$ & $\kappa_{1}\left(\bar{\psi} \psi_{r 2}\right)$ & $\kappa_{2}\left(\bar{\psi} \psi_{r 2}\right)$ \\
\hline This & $16^{3} \times 6$ & $0.0119(4)$ & $0.016(5)$ & $0.0119(4)$ & $0.016(5)$ \\
Work & $24^{3} \times 6$ & $0.0122(4)$ & $0.015(4)$ & $0.0122(4)$ & $0.015(4)$ \\
& $32^{3} \times 8$ & $0.0126(14)$ & $0.014(9)$ & $0.0121(13)$ & $0.012(8)$ \\
{$[23]$} & $16^{3} \times 6$ & $0.0136(3)$ & $0.0133(4)$ & $0.0124(3)$ & $0.0133(5)$ \\
& $24^{3} \times 6$ & $0.0139(3)$ & $0.0150(7)$ & $0.0140(3)$ & $0.0152(7)$ \\
& $32^{3} \times 8$ & $0.0136(3)$ & $0.0142(7)$ & $0.0131(3)$ & $0.0135(7)$ \\
\hline \hline
\end{tabular}

particular case. For this reason, we have performed an additional dedicated study for the $24^{3} \times 6$ lattice, based on new simulations at imaginary chemical potential, to investigate the issue more in deep. Results are reported in the Appendix and show a very good agreement between Taylor expansion and analytic continuation even on coarse lattices, where a very high precision can be achieved in the determination of $\kappa_{1}$ in both cases.

\section{DISCUSSION AND CONCLUSIONS}

Recently, many numerical investigations have been carried out to determine the curvature $\kappa$ of the pseudocritical line in the QCD phase diagram departing from the $\mu_{B}=0$ axis. Estimates obtained by the Taylor expansion technique have been generally lower than those obtained by analytic continuation; however, since the transition is a crossover, care is needed when comparing results obtained by studying different observables.

In this work, the curvature of the pseudocritical line has been studied for $N_{f}=2+1$ QCD, via Taylor expansion and through numerical simulations performed using the tree-level Symanzik gauge action and the stoutsmeared staggered fermion action. This is the same discretization adopted in Refs. [23,24,29]; moreover, we have adopted the same observables and definitions of $\kappa$ investigated in those previous studies, in order to make the comparison closer.

In particular, the location of the phase transition has been determined from the inflection point of the chiral condensate and using two renormalization prescriptions, $\bar{\psi} \psi_{r 1}$ and $\bar{\psi} \psi_{r 2}$, defined, respectively, in Eqs. (7) and (8). The curvature coefficient has been calculated using two different definitions: the first one, $\kappa_{1}$, adopted in Ref. [29], assumes that the value of the renormalized condensate stays constant at the critical temperature as the baryon chemical potential is switched on. The second one, $\kappa_{2}$, which is the same adopted in Refs. [23,24], looks at how the actual inflection point of the condensate moves as a function of $\mu_{B}$ : it is preferable because it is does not rely on particular assumptions; however, it involves the computation of higher-order derivatives, leading to larger numerical uncertainties. 
Our main results can be summarized as follows:

(1) No statistically significant effect due to the renormalization prescription has been observed and finite size effects have been found to be negligible. The values obtained for $\kappa_{2}$ are generally higher than the values obtained for $\kappa_{1}$; however, the difference is well within statistical errors, therefore the two determinations are compatible with our present level of accuracy.

(2) The values obtained for $\kappa_{2}$ are compatible with those obtained in Ref. [23] via analytic continuation; however, present statistics are not enough to determine a reliable estimate for $\kappa_{2}$ via Taylor expansion on finer lattices and to perform an extrapolation to the continuum. Overall, no discrepancy is observed between the results obtained by Taylor expansion and those obtained by analytic continuation also for $\kappa_{1}$ : a possible tension present on the coarsest lattice disappears when the systematics of analytic continuation are properly taken into account (see Appendix).

(3) The final continuum extrapolation that we have given for $\kappa_{1}$ is $\kappa^{\text {cont }}=0.0145(25)$, which is in agreement with results from analytic continuation $[22,23,25]$. In Fig. 7 we report a summary of the most recent determinations of $\kappa$ obtained for QCD at or close to the physical point: the possible tension between analytic continuation and earlier results obtained via Taylor expansion seems to disappear, leaving place to a convergence of the two methods. The preliminary results obtained in Ref. [45] via Taylor expansion

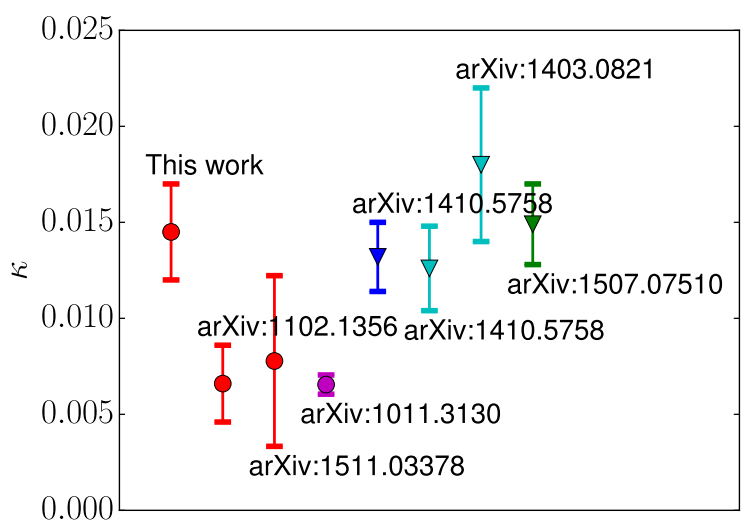

FIG. 7. Curvature coefficient $\kappa$ : comparison with previous determinations. From the left to the right: estimate by this work, Refs. [29,27,28,23,22,25]. Bars marked with circles and triangles indicate that estimates have been obtained, respectively, by Taylor expansion and analytic continuation. More precisely, the red, magenta, blue, cyan and green colors indicate that the estimate has been obtained, respectively, by Taylor expansion + chiral condensate, Taylor expansion + chiral susceptibility, analytic continuation + chiral condensate, analytic continuation + chiral susceptibility and analytic continuation + combined analysis of various observables. with HISQ fermions $(\kappa \sim 0.0120(20))$ point to a similar conclusion.

Regarding the tension (slightly above $2 \sigma$ ) between our present results and the results reported in Ref. [29], where the same discretization, observables and definition of $\kappa_{1}$ were adopted, a possible explanation could be in the different way adopted to take the continuum limit. In our study, for each $N_{t}$, we have determined $\kappa_{1}$ at the corresponding pseudocritical temperature found at $\mu_{B}=0$, and then we have extrapolated those values to the $N_{t} \rightarrow \infty$ limit. In Ref. [29], instead, the definition of $\kappa_{1}$ has been first extended to a wide range of temperatures around $T_{c}$, still based on monitoring how points (temperatures) where the renormalized condensate assumes a fixed value change as a function of $\mu_{B}$; then a continuum extrapolation has been performed over the whole range, thereafter taking the value of this extrapolated function at $T_{c}$.

Two final considerations are in order. First, while a proper extrapolation to the continuum limit for $\kappa_{2}$ is probably out of reach with current computational resources, more statistics would allow us to improve the results and make a closer comparison at least on $N_{t}=6$ lattices. Second, it must be stressed that the convergence towards a common continuum extrapolated value of $\kappa$, which is indicated by recent determinations from analytic continuation and Taylor expansion, is still limited to results obtained only from staggered fermion simulations. It would be important, in the future, to have confirmations also from studies adopting different fermion discretizations.

\section{ACKNOWLEDGMENTS}

Numerical simulations have been performed on the MARCONI and GALILEO machines at CINECA, based on the agreement between Istituto Nazionale di Fisica Nucleare (INFN) and CINECA (under Project No. INF17_npqcd and INF18_npqcd), on the COKA cluster at INFN-Ferrara and at the Scientific Computing Center at INFN-Pisa. F. N. acknowledges financial support from the INFN HPC_HTC project.

\section{APPENDIX: DETAILED COMPARISON IN A SPECIFIC CASE}

This Appendix is dedicated to a more detailed comparison between analytic continuation and Taylor expansion on the $24^{3} \times 6$ lattice, where a tension (at the level of three standard deviations) emerges for $\kappa_{1}$ between the results of the present study and those reported in Ref. [23] (see Table IX).

A possible cause of this tension could be the uncorrect estimate of systematic effects. One of the major sources of systematic errors in analytic continuation is related to the truncation of the polynomial used to fit the observed dependence of $T_{c}$ on the imaginary light quark chemical potential, $\mu_{l}=i \mu_{l, I}$. For small values of the chemical potential, $T_{c}\left(\mu_{l, I}\right)$ is expected to be a purely quadratic function of $\mu_{l, I}$, while higher order corrections are expected to show up at larger chemical potentials, 


$$
T_{c}\left(\mu_{l, I}\right)=T_{c}(0)\left(1+9 \kappa_{1} \hat{\mu}^{2}+c_{4} \hat{\mu}^{4}+O\left(\hat{\mu}^{6}\right)\right),
$$

where $^{2} \hat{\mu} \equiv \mu_{l, I} / T_{c}\left(\mu_{l, I}\right)$. A correct estimate of $\kappa_{1}$ requires us to have higher-order corrections under control; however, in order to do so, one needs a sufficient number of determinations of $T_{c}\left(\mu_{l, I}\right)$ : that was the case in Refs. [23,24] only for $N_{t} \geq 8$ lattices (see in particular the discussion about systematic effects reported in Ref. [24]) but not for $N_{t}=6$, where only two values of the imaginary chemical potential (apart from $\mu_{l, I}=0$ ) were explored, so that a fit with $c_{4} \neq 0$ was not possible.

In Fig. 8, we report a set of determinations of $T_{c}$ for different values of $\hat{\mu}$ obtained on the $24^{3} \times 6$ lattice and adopting the same criterion to locate $T_{c}$ used to define $\kappa_{1}$, i.e., looking at the temperature for which the renormalized chiral condensate (we have considered in particular $\langle\bar{\psi} \psi\rangle_{r 2}$ ) achieves the same value found at $T_{c}(0)$. The black circles reported for the largest values of the chemical potential $(\hat{\mu} / \pi=0.24$ and 0.275$)$ are those already present (apart from $\hat{\mu}=0$ ) in Ref. [23]; the additional determinations at intermediate values of $\hat{\mu}$ are the result of new numerical simulations that we have performed to improve the comparison with Taylor expansion for this particular lattice.

If one tries a best fit according to Eq. (A1) using only the original data of Ref. [23], one is forced to set $c_{4}=0$ (i.e., to neglect quartic corrections) in order to have at least one degree of freedom for the best fit: nevertheless, the quadratic fit is good with a reasonable $\tilde{\chi}^{2} \sim 1$, leading to the unlucky illusion that quartic terms can indeed be neglected and yielding the value $\kappa_{1}=0.0140(3)$ already reported in Table IX).

\footnotetext{
${ }^{2}$ Notice that using $\hat{\mu}$ has an expansion variable instead of $\mu_{l, I}$ leads to different quartic corrections, because $\hat{\mu}$ is normalized by $T_{c}\left(\mu_{l, I}\right)$; however, the quadratic term of the expansion is unchanged.
}

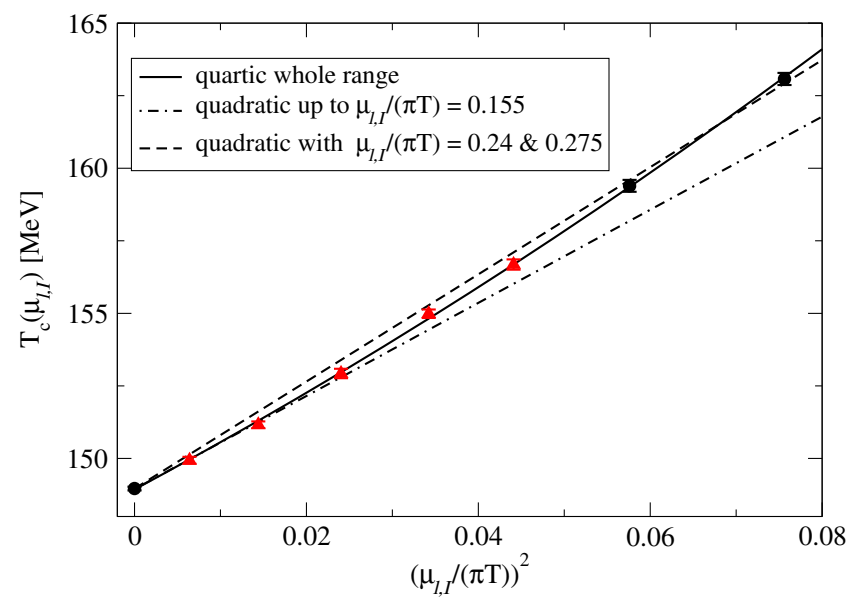

FIG. 8. $T_{c}$ for different values of the imaginary chemical potential obtained on the $24^{3} \times 6$ lattice by looking at the temperature for which the renormalized chiral condensate achieves the same value as for $T=T_{c}(0)$. The black circles $\left(\mu_{l, I} /(\pi T)=0.24\right.$ and 0.275$)$ are those already present in Ref. [23]; the red triangles $\left(\mu_{l, I} /(\pi T)=0.08,0.12,0.155\right.$, 0.185 and 0.21 ) have been obtained by new simulations. The continuous and dashed lines are the results of the best fits described in the text and in the legend.

However, when one considers the whole set of data reported in Fig. 8, a purely quadratic fit works badly $\left(\chi^{2} /\right.$ d.o.f. $\left.\simeq 20 / 6\right)$ and the need to restrict the fit to a range of smaller chemical potentials, or rather to include quartic corrections, emerges clearly. In particular, a purely quadratic fit works well only when considering $\hat{\mu} \leq 0.155$, yielding $\kappa_{1}=0.0121(5)$ with $\chi^{2} /$ d.o.f. $\simeq 1.4 / 2$, while a fit to the whole set of data including quartic corrections leads to $\kappa_{1}=0.0120(5)$ with $\chi^{2} /$ d.o.f. $\simeq 3.5 / 5$. In both cases, the agreement with the Taylor expansion result, $\kappa_{1}=0.0122(4)$, is fully recovered.
[1] M. G. Alford, A. Kapustin, and F. Wilczek, Phys. Rev. D 59, 054502 (1999).

[2] M.-P. Lombardo, Nucl. Phys. B, Proc. Suppl. 83, 375 (2000).

[3] P. de Forcrand and O. Philipsen, Nucl. Phys. B642, 290 (2002); B673, 170 (2003); J. High Energy Phys. 01 (2007) 077; 11 (2008) 012.

[4] M. D'Elia and M. P. Lombardo, Phys. Rev. D 67, 014505 (2003); 70, 074509 (2004).

[5] V. Azcoiti, G. Di Carlo, A. Galante, and V. Laliena, Nucl. Phys. B723, 77 (2005).

[6] H. S. Chen and X. Q. Luo, Phys. Rev. D 72, 034504 (2005).

[7] P. Cea, L. Cosmai, M. D’Elia, and A. Papa, Phys. Rev. D 77, 051501 (2008); 81, 094502 (2010).
[8] L. K. Wu, X. Q. Luo, and H. S. Chen, Phys. Rev. D 76, 034505 (2007).

[9] K. Nagata and A. Nakamura, Phys. Rev. D 83, 114507 (2011).

[10] P. Cea, L. Cosmai, M. D’Elia, C. Manneschi, and A. Papa, Phys. Rev. D 80, 034501 (2009).

[11] A. Alexandru and A. Li, Proc. Sci. LATTICE2013 (2013) 208, arXiv:1312.1201.

[12] P. Cea, L. Cosmai, M. D’Elia, A. Papa, and F. Sanfilippo, Phys. Rev. D 85, 094512 (2012).

[13] C. R. Allton, S. Ejiri, S. J. Hands, O. Kaczmarek, F. Karsch, E. Laermann, C. Schmidt, and L. Scorzato, Phys. Rev. D 66, 074507 (2002). 
[14] C. R. Allton, M. Doring, S. Ejiri, S. J. Hands, O. Kaczmarek, F. Karsch, E. Laermann, and K. Redlich, Phys. Rev. D 71, 054508 (2005).

[15] R. V. Gavai and S. Gupta, Phys. Rev. D 68, 034506 (2003).

[16] R. V. Gavai and S. Gupta, Phys. Rev. D 71, 114014 (2005).

[17] B. J. Schaefer and J. Wambach, Nucl. Phys. A757, 479 (2005).

[18] J. Braun, B. Klein, and B. J. Schaefer, Phys. Lett. B 713, 216 (2012).

[19] C. S. Fischer and J. Luecker, Phys. Lett. B 718, 1036 (2013).

[20] J. M. Pawlowski and F. Rennecke, Phys. Rev. D 90, 076002 (2014).

[21] C. S. Fischer, J. Luecker, and C. A. Welzbacher, Phys. Rev. D 90, 034022 (2014).

[22] P. Cea, L. Cosmai, and A. Papa, Phys. Rev. D 89, 074512 (2014).

[23] C. Bonati, M. D’Elia, M. Mariti, M. Mesiti, F. Negro, and F. Sanfilippo, Phys. Rev. D 90, 114025 (2014).

[24] C. Bonati, M. D'Elia, M. Mariti, M. Mesiti, F. Negro, and F. Sanfilippo, Phys. Rev. D 92, 054503 (2015).

[25] R. Bellwied, S. Borsanyi, Z. Fodor, J. Günther, S. D. Katz, C. Ratti, and K. K. Szabo, Phys. Lett. B 751, 559 (2015).

[26] P. Cea, L. Cosmai, and A. Papa, Phys. Rev. D 93, 014507 (2016).

[27] P. Hegde, H.-T. Ding (Bielefeld-BNL-CCNU Collaboration), Proc. Sci. LATTICE2016 (2016) 141, arXiv:1511 .03378 .

[28] O. Kaczmarek, F. Karsch, E. Laermann, C. Miao, S. Mukherjee, P. Petreczky, C. Schmidt, W. Soeldner, and W. Unger, Phys. Rev. D 83, 014504 (2011).

[29] G. Endrodi, Z. Fodor, S. D. Katz, and K. K. Szabo, J. High Energy Phys. 04 (2011) 001.

[30] P. Weisz, Nucl. Phys. B212, 1 (1983).

[31] G. Curci, P. Menotti, and G. Paffuti, Phys. Lett. B 130, 205 (1983); 135, 516(E) (1984).
[32] C. Morningstar and M. J. Peardon, Phys. Rev. D 69, 054501 (2004).

[33] A. Bazavov, D. Toussaint, C. Bernard, J. Laiho, C. DeTar, L. Levkova, M. B. Oktay, and S. Gottlieb et al., Rev. Mod. Phys. 82, 1349 (2010).

[34] Y. Aoki, S. Borsanyi, S. Durr, Z. Fodor, S. D. Katz, S. Krieg, and K. K. Szabo, J. High Energy Phys. 06 (2009) 088.

[35] S. Borsanyi, G. Endrodi, Z. Fodor, A. Jakovac, S. D. Katz, S. Krieg, C. Ratti, and K. K. Szabo, J. High Energy Phys. 11 (2010) 077; S. Borsanyi, Z. Fodor, C. Hoelbling, S. D. Katz, S. Krieg, and K. K. Szabo, Phys. Lett. B 730, 99 (2014).

[36] M. Cheng, N. H. Christ, S. Datta, J. van der Heide, C. Jung, F. Karsch, O. Kaczmarek, and E. Laermann et al., Phys. Rev. D 77, 014511 (2008).

[37] E. Laermann, F. Meyer, and M. P. Lombardo, J. Phys. Conf. Ser. 432, 012016 (2013).

[38] S. Ejiri, F. Karsch, E. Laermann, C. Miao, S. Mukherjee, P. Petreczky, C. Schmidt, W. Soeldner, and W. Unger, Phys. Rev. D 80, 094505 (2009).

[39] M. A. Clark, A. D. Kennedy, and Z. Sroczynski, Nucl. Phys. B, Proc. Suppl. 140, 835 (2005).

[40] M. A. Clark and A. D. Kennedy, Phys. Rev. D 75, 011502 (2007).

[41] M. A. Clark and A. D. Kennedy, Phys. Rev. Lett. 98, 051601 (2007).

[42] C. Bonati, S. Coscetti, M. D'Elia, M. Mesiti, F. Negro, E. Calore, S. F. Schifano, G. Silvi, and R. Tripiccione, Int. J. Mod. Phys. C 28, 1750063 (2017).

[43] C. Bonati, E. Calore, M. D'Elia, M. Mesiti, F. Negro, F. Sanfilippo, S. F. Schifano, G. Silvi, and R. Tripiccione, Int. J. Mod. Phys. C 29, 1850010 (2018).

[44] C. Bonati, G. Cossu, M. D’Elia, and P. Incardona, Comput. Phys. Commun. 183, 853 (2012).

[45] P. Steinbrecher, arXiv:1807.05607. 